\title{
EFEITO DO MANEJO DO LENÇOL FREÁTICO NA ADAPTAÇÃO FISIOMORFOLÓGICA DE DUAS ESPÉCIES DE TRIGO AO ENCHARCAMENTO ${ }^{1}$
}

\author{
Rinaldo de O. Calheiros ${ }^{2}$, Décio E. Cruciani ${ }^{3}$, Flávio B. Arruda ${ }^{2}$, Rachel B. Queiroz Voltan ${ }^{4}$, \\ Emílio Sakai ${ }^{2} \&$ Regina Célia de M. Pires ${ }^{2}$

\section{RESUMO}

Estudou-se o efeito de três manejos do lençol freático na indução de adaptações fisiomorfológicas dos trigos Triticum aestivum L. e Triticum durum L. à hipoxia, caracterizando e inferindo a influência relativa dos principais fatores físicos e biológicos interferentes. $\mathrm{O}$ experimento foi conduzido na ESALQ/USP, Piracicaba, SP, simulando-se ao máximo um meio físico/condição natural de uma várzea. Após a indução na fase vegetativa, através de períodos hipóxicos com duração progressiva, o grau de adaptação foi avaliado através de inundação permanente, incluindo o florescimento/formação de grãos. Observou-se: resposta diferenciada de exigência e/ou capacidade de extração de nutrientes entre as duas espécies; que a adubação foliar foi ineficiente para suprir as deficiências nutricionais da planta; que os manejos com curtos períodos de hipoxia induziram o trigo a adaptações fisiomorfológicas, porém não na intensidade ou eficiência de que resultassem parâmetros de produção em níveis adequados; enfim, que o manejo com o lençol freático mantido a $15 \mathrm{~cm}$ de profundidade durante todo o ciclo cultural foi o que propiciou melhor desempenho do trigo em cultivo sob encharcamento.

Palavras-chave: drenagem, manejo, hipoxia, tolerância

\section{EFFECT OF THE WATER TABLE MANAGEMENT IN THE MORPHO-PHYSIOLOGICAL ADAPTATION OF TWO WHEAT SPECIES TO WATERLOGGING}

\begin{abstract}
The effect of three different water table managements in the morpho-physiological adaptation to waterlogging of Triticum aestivum, L. and Triticum durum, L., and the relative influence of the main physical and biological interferance factors were studied. The trial was conducted at ESALQ/USP, Piracicaba, SP, under simulated conditions of a natural low land. After introduction of stress adaptation during the vegetative growth stage, while applying progressive times of hipoxics periods, there were observed different requirements and/or extraction capacities of nutrients between the two species; the fertilization on leaves was not enough to avoid nutritional deficiencies in wheat under hipoxia. The water table management used resulted in morpho-physiological adaptations, but not enough to reach adequate biometric parameters of productivity. The water table at a $15 \mathrm{~cm}$ depth throughout the crop cycle resulted in better performance.
\end{abstract}

Key words: drainage, management, hipoxia, tolerance

\footnotetext{
Recebido em 10/01/2000, Protocolo 002/00

${ }^{1}$ Parte da tese de doutorado do primeiro autor na ESALQ/USP

${ }^{2}$ Pesquisador do Instituto Agronômico, IAC, CP 28, CEP 13.001 - 970, Campinas, SP. Fone: (0xx19) 241 5188, Fax: (0xx19) 2314943. E-mail: rocalhei@cec.iac.br, farruda@cec.iac.br,emilio@cec.iac.brercmpires@cec.iac.br

${ }^{3}$ Professor Titular, Departamento de Engenharia Rural, ESALQ/USP. Fone: (0xx19) 429 4217, Fax: (0xx19) 4330934. E-mail: cruciani@carpa.ciagri.usp.br

${ }^{4}$ Pesquisadora do Instituto Agronômico, IAC, CP 28, CEP 13.001 - 970, Campinas, SP. Fone: (019) 231 5422, Fax: (0xx19) 2314943. E-mail:rachelqv@barao.iac.br
} 


\section{INTRODUÇÃO}

Em terras baixas, a monocultura é problema mundial (Garrity $\&$ Pernito, 1996); assim, a cultura do trigo poderia desempenhar papel muito importante como alternativa de sucessão ou rotação ao arroz, em que o sucesso seria altamente desejável para atender a extensas áreas definitivamente comprometidas pela infestação de plantas invasoras, em especial capim arroz e arroz vermelho.

A diversificação de culturas em terras baixas, no entanto, é fortemente caracterizada como de alto risco de encharcamento ocasional, exigindo a prática da drenagem, normalmente exagerada, levando à degradação do meio. Como solução desse impasse surgem os trabalhos de tolerância fisiomorfológica das culturas ao encharcamento, cujos resultados são muito variáveis em função da diversidade de fatores interferentes.

Dentre as culturas, o trigo encontra-se em condição intermediária de suscetibilidade à hipoxia hídrica sendo, no entanto, uma das mais sensíveis dentre as gramíneas, fato este associado, segundo Waters et al. (1991), a uma relativamente mais baixa taxa de fermentação alcoólica que do arroz e do milho.

É escassa a literatura sobre trigo sob encharcamento, sendo ainda maior se considerado para as condições tropicais, conduzidas sob condições naturais, durante todo o ciclo, com os principais fatores interferentes monitorados. A maioria dos trabalhos é de laboratório, com substrato artificial e/ou esterilizado (van Wadman \& van Andel, 1985; Huang et al., 1994), com plântulas (Minchin \& Summerfield, 1976; Trought \& Drew, 1980; Waters et al., 1991) e/ou sem a adequada caracterização ou monitoramento do solo/clima/água (Singh et al., 1991; White \& Molano, 1994; Garrity \& Pernito, 1996; Umaharan et al., 1997).

A influência do ambiente é tão grande que os trabalhos realizados em clima temperado devem ser interpretados com muito cuidado, dentre outras razões pela baixa demanda transpirométrica e em razão do encharcamento poder encontrar a planta em dormência. Beard \& Martin (1970) afirmam que forrageiras em dormência ou semi-dormência toleram períodos mais longos de inundação que sob desenvolvimento ativo.

Nas regiões tropicais e subtropicais, a temperatura maximiza o efeito hipóxico tanto pela menor dissolução do $\mathrm{O}_{2}$ na água como pelo maior consumo da planta e microrganismos, implicando no aumento da velocidade das reações químicas.

Sabe-se que a melhor indicadora de disponibilidade de oxigênio do solo para a planta é a TDO (Taxa de Difusão do Oxigênio) que correlacionada a várias respostas da planta (Sojka \& Soltzy, 1980) varia mais freqüentemente dentro da faixa de 0 a $120.10^{-8} \mathrm{~g} \mathrm{~cm}^{-2} \mathrm{~min}^{-1}$ (Glinski \& Stepiewski, 1986). Silverbush et al. (1979) apontam que a densidade de raízes de trigo começa a reduzir-se a uma TDO de $30.10^{-8} \mathrm{~g} \mathrm{~cm}^{-2} \mathrm{~min}^{-1} \mathrm{e}$ cai abruptamente para menos de $1 / 5$ do número original, no intervalo de 30 a $24.10^{-8} \mathrm{~g} \mathrm{~cm}^{-2} \mathrm{~min}^{-1}$, o primeiro aceito como limite para o desenvolvimento da raiz e o segundo como crítico.

Como efeitos decorrentes, surgem modificações de natureza física, como a penetração das raízes e a permeabilidade do solo, nutricionais, químicas e biológicas.

Teoricamente, pode ser factível induzir-se a adaptabilidade fisiomorfológica das plantas através de um regime de hipoxia/ anoxia específico, sendo que o manejo adequado do lençol freático pode ser a chave para este processo. O estabelecimento abrupto do estado hipóxico não permite aclimatação e/ou a indução de um metabolismo alternativo antes da condição se tornar letal (van Wadman \& van Andel, 1985). Por outro lado, há um consenso de que a anoxia atingida de forma gradativa, passando por estágios intermediários de hipoxia, exerce grande importância na adaptação da planta sob encharcamento. Johnson et al. (1989) reportam que a pré-exposição à hipoxia resultou em aumento da atividade da ADH, da carga energética de adenina e da sobrevivência do milho. Wignarajah \& Greenway (1976) apontam que em cevada e milho concentrações intermediárias de $\mathrm{O}_{2}(3-13 \%)$ produziram mais estímulo da atividade $\mathrm{ADH}$ que a baixas concentrações $(<3 \%)$. Morfologicamente, o início do processo parece estar ligado ao papel dos fito-hormônios, destacando-se o etileno (Schaffer et al., 1992) induzindo à epinastia foliar, ao crescimento de raízes adventícias, à hipertrofia dos caules e à produção de aerênquima em certas espécies de plantas (Bradford \& Yang, 1981; Kawase, 1981).

O desenvolvimento de raízes adventícias tem o propósito de facilitar a absorção de $\mathrm{O}_{2}$, sendo a elas creditada uma condutância estomática perto da normal (Sena Gomes \& Kozlowski, 1980). A habilidade para sua formação é considerada, geralmente, o maior fator de tolerância da plantas florestais (Hook \& Brown, 1973).

O hipertrofiamento das lenticelas é caracterizado pelo alargamento celular, numa forma mais esférica e pelo desaparecimento de sua película de fechamento; juntamente com o aerênquima, funcionam como órgãos de aumento da absorção de $\mathrm{O}_{2}$ e transporte para as raízes (Kawase, 1981).

Com base nas considerações acima, há fortes evidências de que o manejo adequado do lençol freático pode ser a melhor estratégia para a exploração de culturas mesófitas em terras baixas, proporcionando às plantas maiores chances de sobrevivência, quando da ocorrência de um evento hipóxico temporal como, por exemplo, uma enchente resultante de uma chuva à montante.

Inquestionável é que um entendimento maior da resposta final do trigo à hipoxia só é permitido se desenvolvido em condições naturais, com adequada e sistemática caracterização e monitoramento dos principais fatores interferentes.

O objetivo deste trabalho foi: estudar o efeito de diferentes manejos do lençol freático nos principais mecanismos adaptativos/mitigadores da hipoxia utilizada pelo trigo, caracterizar e monitorar os principais fatores de produção interferentes numa condição de hipoxia, interpretando seu papel individual e interativo na resposta final da cultura.

\section{MATERIAL E MÉTODOS}

No desenvolvimento do trabalho, procurou-se simular, o máximo possível, a condição natural de uma área de terras baixas produtivas.

Constituíram-se, como parcela experimental, caixas de cimento amianto de $1000 \mathrm{~L}$, com área de $1,9 \mathrm{~m}^{2}$. O lençol freático foi manejado por meio de tubos de duas polegadas, posicionados na base interna das caixas-parcela, conectados a um sistema de vasos comunicantes, e a um recipiente adjacente cheio de água, contendo uma bóia hidráulica que exerceu o controle e manutenção do nível da água (no recipiente) e do lençol freático (na caixa-parcela). $\mathrm{O}$ monitoramento do nível do 
Tabela 1. Análise química do solo utilizado no estudo

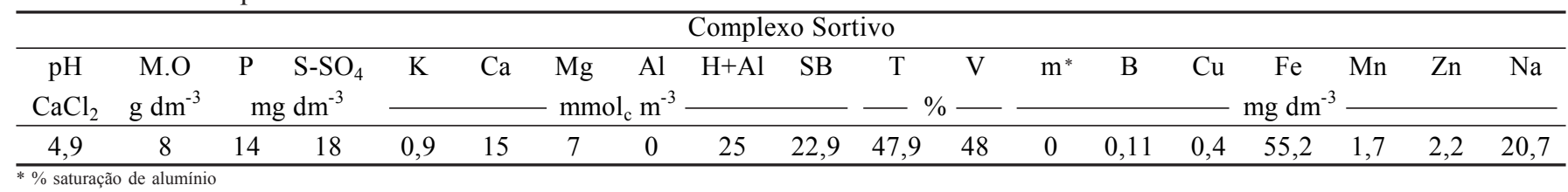

lençol foi executado por leitura diária de poço de observação, construído no solo e revestido por tubo de PVC de duas polegadas, perfurado.

O substrato foi composto de uma camada de pedra brita 1, na altura dos drenos $(10 \mathrm{~cm})$, seguida de outra camada de mesma espessura de pedrisco, com $60 \mathrm{~cm}$ de terra retirada de uma várzea de solo Podzólico Vermelho-Amarelo abrupto, A moderado e espesso, textura arenosa, unidade Serrinha (Arenic Abruptic Paleudalt), ácido, de média fertilidade.

As características físico-hídricas do solo são detalhadamente, apresentadas em Calheiros (2000).

As análises químicas do solo e da água de rio (não tratada), utilizada para formar e manter o lençol freático dentro das caixasparcela, são apresentadas nas Tabelas 1 e 2; nesta, os teores encontrados foram comparados, em parte, com os valores máximos permitidos para consumo humano e, em parte, com os teores máximos permitidos para irrigação.

Tabela 2. Análise da água utilizada no estudo

\begin{tabular}{|c|c|c|}
\hline Parâmetro & $\begin{array}{l}\text { Valor } \\
\mathrm{mg} \mathrm{L}^{-1}\end{array}$ & $\begin{array}{l}\text { VMPC } \\
\mathrm{mg} \mathrm{L}^{-1}\end{array}$ \\
\hline Nitrogênio & 0 & \\
\hline Sulfato & 0,8 & $961,0 *$ \\
\hline Nitrato & 0 & $10,0 *$ \\
\hline Cloreto & 1,0 & $1050,0 *$ \\
\hline Fósforo & 0,01 & $2,0 *$ \\
\hline Potássio & 0,4 & $2,0 *$ \\
\hline Cálcio & 8,5 & $400,0 *$ \\
\hline Magnésio & 7,5 & $60,7^{*}$ \\
\hline Ferro & 0,4 & $0,3 *$ \\
\hline Alcalinidade & 36,2 & \\
\hline Zinco & 0 & $5,0 *$ \\
\hline Sódio & 0,7 & $920,0 *$ \\
\hline Cobre & 0 & 1,0 \\
\hline Manganês & 0 & 0,1 \\
\hline Dureza & 52,1 & 500,0 \\
\hline Acidez & 18,9 & \\
\hline Gás carbônico & 8,7 & \\
\hline Sedimentos & 1,7 & \\
\hline Turbidez & 11,0 & 1,0 \\
\hline $\mathrm{pH}$ & 6,8 & $6,0-8,5^{*}$ \\
\hline
\end{tabular}

Utilizou-se o Triticum aestivum, $L$. cultivar IAC 24, destinado à panificação, e o Triticum durum, L. cultivar IAC 1003, destinado à produção de massas finas, plantadas em 17/05/98, na população de 450 plantas $\mathrm{m}^{-2}$, no espaçamento de $20 \mathrm{~cm}$ entre linhas.

O delineamento experimental foi de parcelas subdivididas, inteiramente ao acaso, utilizando-se os seguintes tratamentos, em cinco repetições: $T_{1}$ : testemunha; $T_{2}$ : com elevação e manutenção permanente do lençol freático (LF) a $15 \mathrm{~cm}$ de profundidade, até a maturação fisiológica da cultura; $\mathrm{T}_{3}$ : elevação e manutenção do LF a $15 \mathrm{~cm}$; aplicação de períodos progressivos de adaptação induzida (PPAI) de 2, 4, 24 h, nos intervalos de 15 dias; aplicação do teste de adaptação à hipoxia (TAH) até a maturação fisiológica da cultura e; $\mathrm{T}_{4}$ : igual a $\mathrm{T}_{3}$, com intervalo, entre as PPAIs, de 7 dias.

Até o início do perfilhamento, todos os tratamentos foram conduzidos sob normoxia, mantendo-se a umidade do solo na capacidade de campo quando, então, foram diferenciados. $\mathrm{O} \mathrm{T}_{1}$, teoricamente, representou o potencial máximo produtivo relativo da cultura neste solo e clima conduzido permanentemente em solo aerado, recebendo irrigações subterrâneas.

Os PPAIs constituíram-se curtos períodos de encharcamento do solo, através de elevações do LF a $1 \mathrm{~cm}$ acima da superfície do solo, com seqüencial rebaixamento de nível, de $15 \mathrm{~cm}$, enquanto o TAH se constitui da elevação definitiva do LF a $1 \mathrm{~cm}$ acima da superfície do solo, com seu rebaixamento completo ocorrido apenas na maturação fisiológica da cultura. A quantificação da eficiência dos manejos na indução adaptativa da cultura à hipoxia foi realizada de modo comparativo entre tratamentos, através dos parâmetros biométricos do desempenho da cultura. A adubação de plantio foi com base na análise química, o nitrogênio foi aplicado parceladamente, na forma de uréia e se executaram adubações foliares sempre em resposta a sintomas de deficiência nutricional, objetivando-se minimizar possível deficiência no processo de absorção pelas raízes sob hipoxia. Utilizaram-se dois tipos de adubo foliar, um contendo $8 \%$ de cálcio e $2 \%$ de boro e o outro de formulação 14-4-6, contendo ainda $0,8 \%$ de enxofre, $1,5 \%$ de manganês; $2 \%$ de zinco, $1,5 \%$ de magnésio, $0,1 \%$ de boro e $0,05 \%$ de molibdênio, ambos aplicados na dosagem correspondente a $3 \mathrm{~L} \mathrm{ha}^{-1}$.

A deficiência nutricional das plantas foi avaliada por análise foliar aos 40 DAE (dias após emergência) coletando-se, em 2 plantas por cultivar, por repetição, 2 folhas de cima, verdes, e 2 de baixo, com até $40 \%$ de sua superfície amarelada. Avaliou-se a taxa de difusão de oxigênio no solo (TDO) e o potencial redox, utilizando-se aparelho desenvolvido pelo CNPDIA/Embrapa (Herrmann et al., 1997). Avaliou-se a taxa de liberação de etileno pelas raízes, coletando-se seus ápices no comprimento de $3 \mathrm{~cm}$, acondicionados em frasco especial e executada leitura após 2 dias de descanso, utilizando-se Detector de Ionização de Chama.

As transformações morfológicas foram caracterizadas através de observações, com lupa, da presença de lenticelas e de raízes adventícias.

Os parâmetros biométricos de produção na colheita foram avaliados utilizando-se 10 plantas por repetição e o rendimento de grãos o foi através da amostragem em uma fileira central de $1,0 \mathrm{~m}$ de comprimento, enquanto os dados foram analisados estatisticamente, através da análise de variância $(\mathrm{P}<0,05)$, teste de significância de Duncan a $5 \%$ e determinação do Erro-Padrão.

\section{RESULTADOS E DISCUSSÃO}

\section{Eventos climáticos e fenologia da cultura}

$\mathrm{Na}$ seqüência de eventos climáticos, fenológicos e de condução da cultura (Figura 1) observa-se que a temperatura 
(A)

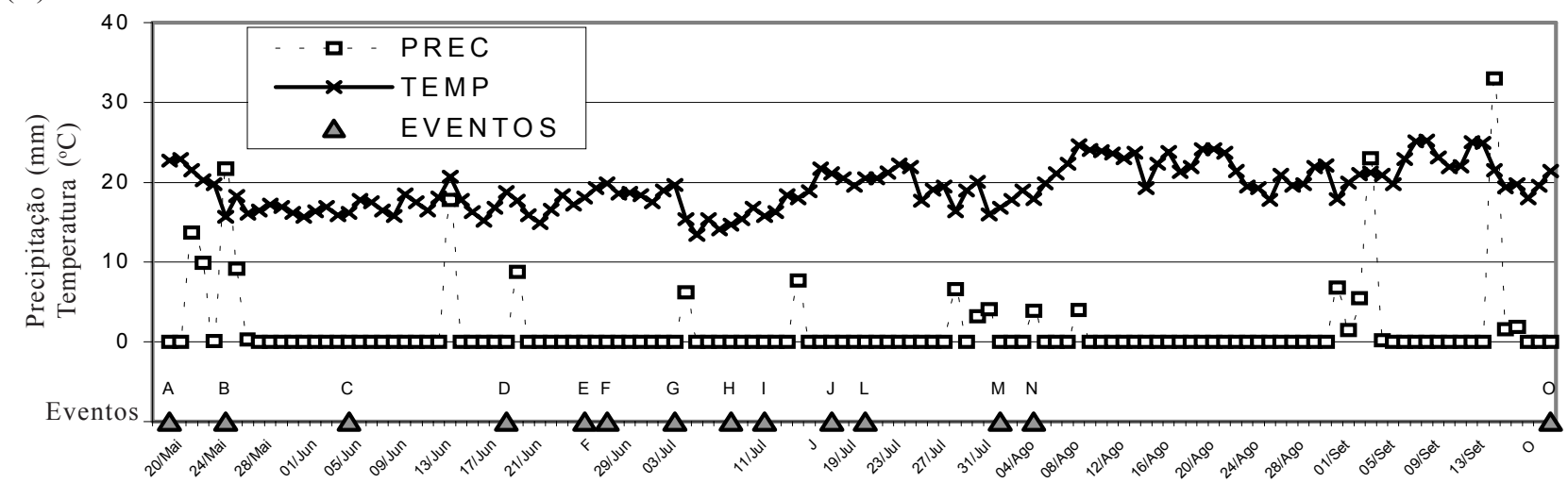

(B)

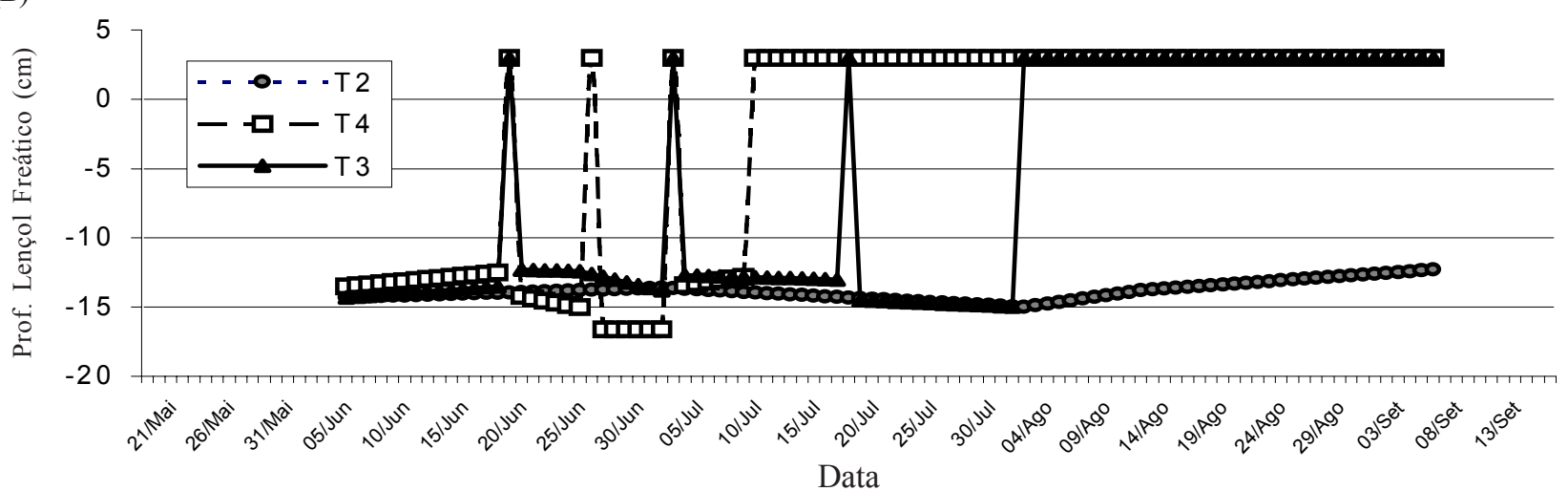

Legenda, eventos e algumas observações

A Plantio

B 5 DAE (dias após emergência), Emergência

C 11 DAE, elevação LF $15 \mathrm{~cm} ; 12$ DAE; irrigação no T

D $26 \mathrm{DAE}^{\text {a }}$ PPAI no $\mathrm{T}_{3}$ e $\mathrm{T}_{4}$, pls. com 4 folhas, pleno perfilha/to, altura de planta $15 \mathrm{~cm}$, sem diferença fenológica

E 33 DAE, $2^{\text {a }}$ PPAI no $\mathrm{T}_{4}$

F 35 DAE, primeiros sintomas hipóxicos, amarelecimento folhas de baixo (FBA) no $T_{3}$ e $T_{4}$; irrigação no $T_{1}-36$ DAE, $1^{\text {a }}$ pulverização foliar

G 40 DAE, $3^{a}$ PPAI no $T$, 2a PPAI no $T$ - clorose somente no $T$, 43 DAE, 30 a $50 \%$ das plantas com FBA nos $T$ e $T$, clorose no $T$; $T$ sem FBA, com ligeira clorose

$\mathrm{H} 46 \mathrm{DAE}$, T. aestivum com folha bandeira estendida, início do emborrachamento em todos os tratamentos; T. durum no final da alongação/início do emborrachamento; no $\mathrm{T}_{4}$ T. aestivum c/ $30-40 \mathrm{~cm}$ e T. durum com $25 / 30 \mathrm{~cm}$ de altura; - $47 \mathrm{DAE}$, inicio TAH no $\mathrm{T}_{4}$

I $49 \mathrm{DAE}, \mathrm{T}_{2}$ com clorose, porém com menor sintoma de hipoxia que $\mathrm{T}_{3}$ e $\mathrm{T}_{4}$, sem diferença de sintoma de hipoxia entre cvs. em qualquer tratamento

J 55 DAE, $3^{\circ}$ PPAI no $T$

L 58 DAE, $2^{\mathrm{a}}$ pulverização foliar; irrigação do $\mathrm{T}_{1}$;

M 68 DAE, - início TAH no $\mathrm{T}$; $\mathrm{T}$ a 21 dias de TAH

N 73 DAE, - Espigamento (\%) do T. aestivum e T. durum, respectivamente: $\mathrm{T}_{1}$ - as duas cvs. $100 \%$; $\mathrm{T}_{2}-90 \%$ e $5 \%, \mathrm{~T}_{3}-80 \%$ e $5 \%$; $\mathrm{T}_{4}-50 \%$, e $2 \%$; no $\mathrm{T}_{4}$ claro sintoma no T. aestivum, com algumas plantas secas; as demais condições (cultivar e tratamento) com bom desenvolvimento; as duas cvs., em todos os tratamentos, com folhas de cima verdes e as de baixo secas. - 80 DAE; irrigação do $\mathrm{T}_{1}$

O 119 DAE, - colheita

Figura 1. Seqüência de eventos (A) precipitação $(\mathrm{mm})$ e temperatura média $\left({ }^{\circ} \mathrm{C}\right)$ e $(B)$ flutuação do L. F. ocorridos durante o ciclo fenológico

oscilou em torno de $18^{\circ} \mathrm{C}$ durante o estádio vegetativo. Coincidentemente, a partir da implantação do TAH no $\mathrm{T}_{4}$, elevou-se $\mathrm{e}$, seqüencialmente, ainda mais na submissão do $\mathrm{T}_{3}$ ao TAH.

$\mathrm{O}$ fato das temperaturas se elevarem a partir da implantação dos TAHs, tornou esse período ainda mais difícil para as plantas, por essa ocorrência justamente no estádio de intensa atividade metabólica, contando com um sistema radicular pouco efetivo, tendo ainda que satisfazer a uma demanda evapotranspirométrica mais elevada (Figura 1A).

A manifestação idêntica dos primeiros efeitos da hipoxia no $\mathrm{T}_{3}$ e no $\mathrm{T}_{4}$, aos $35 \mathrm{DAE}$ - amarelecimento das folhas de baixo, e nenhum sintoma no $T_{2}$, podem ser interpretados mais como efeito da prática do período curto de encharcamento, conjugado com o lençol elevado a $15 \mathrm{~cm}$, que da intensidade das PPAIs ministradas. Dentro de uma gama de possibilidades infere-se que, com o aumento da evapotranspiração, o sistema radicular, já então mais fortemente afetado pela PPAI e limitado pelo $L F$ a $15 \mathrm{~cm}$ de profundidade, não conseguiu suprir a planta, de forma conveniente, o que foi agravado ainda mais pela temperatura, então na faixa de $20^{\circ} \mathrm{C}$, atipicamente alta para esta época do ano. Dentre as opções de indução adaptativa, talvez a mais prática e efetiva estratégia seja induzir o sistema radicular à adaptação morfológica proporcionalmente com a velocidade do aumento da demanda evapotranspirométrica, o que talvez possa ser conseguido através da elevação gradativa do LF, ao invés de curtos e intermitentes períodos hipóxicos, como o empregado.

Fenologicamente, aos 46 DAE o T. durum apresentava-se um pouco mais atrasado atribuindo-se exclusivamente ao efeito genético, atestado pela condição idêntica mostrada no $T_{1}$. Quanto ao sintoma de hipoxia, porém, não havia diferença entre as duas espécies (Figura 2).

Aos 73 DAE e a despeito do ciclo fenológico mais avançado do T. aestivum, entre os tratamentos, no T. aestivum, havia um gradiente com o grau de encharcamento $(100,90,80$ e $50 \%$ de espigamento para $\mathrm{T}_{1}, \mathrm{~T}_{2}, \mathrm{~T}_{3} \mathrm{e} \mathrm{T}_{4}$, respectivamente) inferindo-se haver retardamento no ciclo cultural com a hipoxia. No T. durum, pelo fato da testemunha estar a $100 \%$ de espigamento e todos 


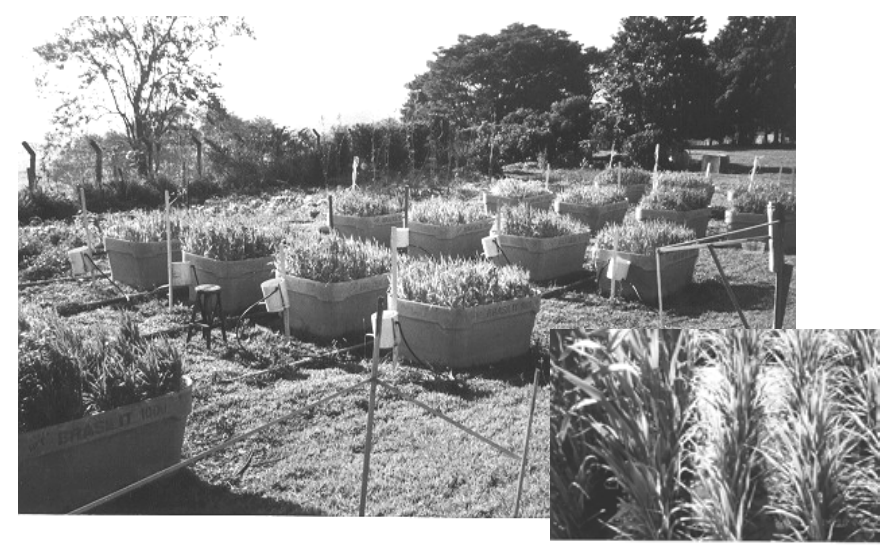

Figura 2. Desenvolvimento fenológico das duas espécies de trigo, quando da aplicação do $\mathrm{TAH}$ no $\mathrm{T}_{4}$. Vista geral do experimento e detalhe da lâmina de inundação

os outros tratamentos numa porcentagem igual e menor que $10 \%$, infere-se que, a despeito de ser mais tardio, o efeito da hipoxia no ciclo foi ainda maior e sem distinção entre as intensidades com que elas foram implementadas; assim, mesmo com as possíveis adaptações fisiomorfológicas das plantas à hipoxia, a partir do emborrachamento a inundação permanente passa a afetar muito.

\section{Deficiências nutricionais $\mathbf{x}$ adubação foliar}

A análise química do solo revelou-o de baixa fertilidade, apresentando baixos teores de matéria orgânica, potássio, fósforo e boro; médios de magnésio, cobre e manganês, e alto de cálcio, sulfato, ferro e zinco. A análise da água revelou-a de boa qualidade, sem limitação alguma para prática de irrigação.

O amarelecimento das folhas inferiores motivou a realização da primeira pulverização de adubo foliar aos 36 DAE. Aos 43 DAE os $\mathrm{T}_{3}$ e $\mathrm{T}_{4}$ manifestavam acentuada clorose. $\mathrm{O}$ mesmo

T. aestivum
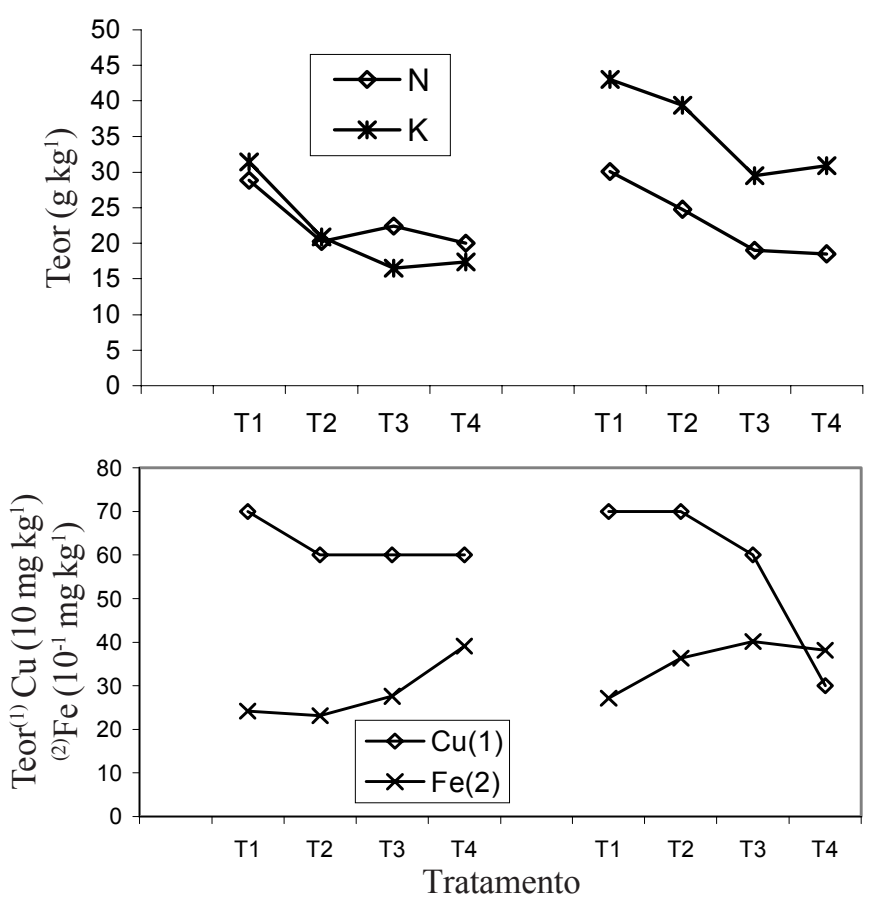

ocorre com o $\mathrm{T}_{2}$, porém em menor intensidade sem, no entanto, apresentar amarelecimento das folhas inferiores e assim, além do efeito do estresse hídrico, a deficiência nutricional passou a ser preocupante, levando à hipótese de que talvez a aplicação de adubo foliar tivesse sido ineficiente.

A análise foliar (Figura 3) revelou, nas duas espécies, tendência ao decréscimo gradativo dos teores de nitrogênio e potássio, com a intensidade de inundação. O mesmo ocorreu com o fósforo no $T$. aestivum e o enxofre no T. durum. Em todas, no entanto, a proximidade dos valores entre os $\mathrm{T}_{3}$ e $\mathrm{T}_{4}$ indica que mesmo os PPAIs a nível de 2 e $4 \mathrm{~h}$, no intervalo de 15 dias, já foram suficientes para limitar suas presenças na folha.

Esses resultados são coerentes com os aspectos físicos observados, mais discretos no $\mathrm{T}_{2}$ e mais evidentes nos $\mathrm{T}_{3}$ e $\mathrm{T}_{4}$. Segundo Glinski \& Stepniewski (1986) a anoxia causa decréscimo no conteúdo de potássio em todas as partes da planta. Eles afirmam que no trigo o encharcamento inibe completamente a sua absorção com o deslocamento do nutriente das folhas velhas para as jovens, perceptível após 2 dias de encharcamento; apontam, ainda, que a deficiência em potássio é o sintoma mais sensível ao encharcamento, visto primeiro e mais distintamente que no caso dos outros íons. Como os níveis de $\mathrm{K}$ encontrados no $T$. durum foram, na média dos tratamentos e no $\mathrm{T}_{1}, 30 \%$ maiores que no $T$. aestivum, pode-se concluir que o T. durum é mais extrativo ou exigente nesse elemento, que o T. aestivum.

Apenas no T. durum o teor de fósforo se elevou do $\mathrm{T}_{1}$ para o $\mathrm{T}_{2}$, como que favorecido pelo maior conteúdo de umidade do solo, seguindo-se, no entanto, um decréscimo abrupto e proporcional com a intensidade de inundações periódicas. Um decréscimo da retirada de fósforo pelo trigo sob encharcamento é apontado por Labanauska et al. (1975).

T. aestivum

T. durum
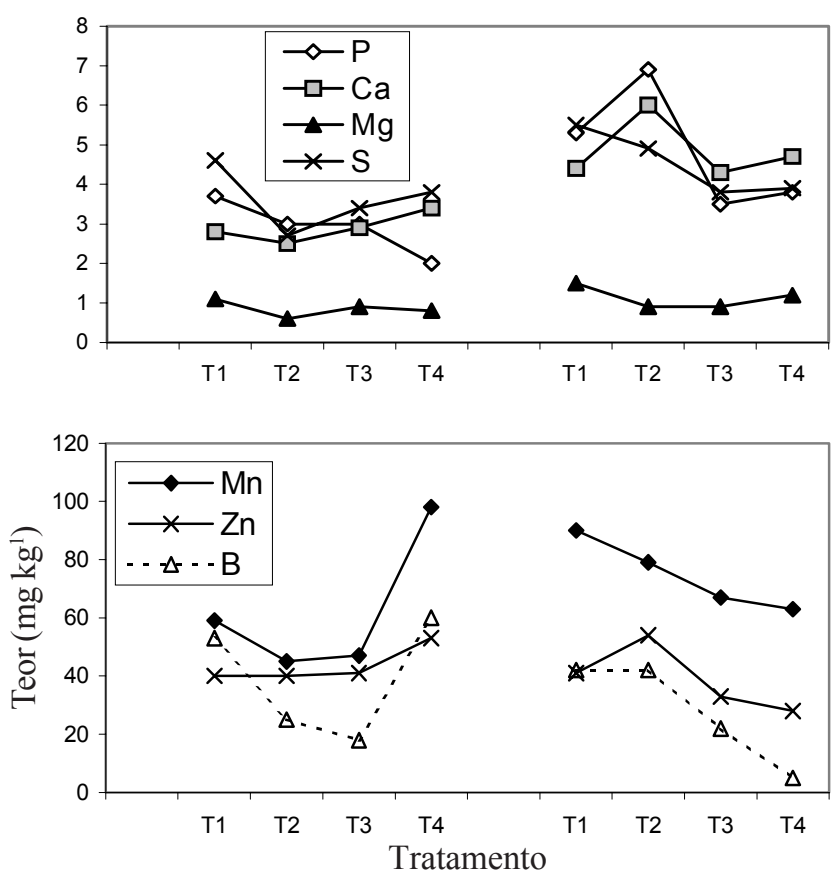

Figura 3. Teor de macro e micronutrientes nas folhas (matéria seca) do T. aestivum e do T. durum 
Segundo Glinski \& Stepiewski (1986) um decréscimo de fósforo na planta aparece após a concentração de oxigênio cair abaixo de $0,05 \mathrm{~m}^{3} \mathrm{~m}^{-3}$. Conforme Trought \& Drew (1980) quando raízes de trigo foram submersas, a retirada de fósforo foi quase completamente paralisada, a despeito do crescimento das plantas continuar mais lentamente; após 2 dias, foram diagnosticados decréscimo do conteúdo de fósforo nas folhas velhas e acréscimo nas jovens e, após 2 semanas, mais de $80 \%$ do fósforo tinham sido deslocados das folhas velhas para as jovens. Essas observações são coerentes com o comportamento do conteúdo de fósforo obtido neste trabalho. É importante enfatizar que a translocação dos nutrientes $\mathrm{N}, \mathrm{P}$ e K das folhas de baixo para as de cima, induzida pela hipoxia, referenda o acerto na metodologia de coleta utilizada $-50 \%$ de folhas velhas de baixo e $50 \%$ de folhas novas de cima.

O teor de Ca no T. aestivum apresentou tendência de elevação gradativa com a hipoxia. Letey et al. (1961) afirmam que o conteúdo de cálcio, assim como o do magnésio, parece ser menos afetado pela anoxia que o nitrogênio, potássio e fósforo. De acordo com Trought \& Drew (1980) o trigo não manifesta qualquer tendência ao deslocamento desses dois elementos das folhas velhas para as novas. Nas curvas (Fig. 3), nota-se que, com exceção dos níveis atingidos no $\mathrm{T}_{2}$ pelo $T$. durum, há tendência, nas duas espécies, a exemplo do observado com o magnésio, à pouca modificação dos teores, o que mostra ser o comportamento desses trigos, em nossas condições, coerente com a literatura. Trabalhos futuros poderão elucidar melhor este comportamento. No caso do enxofre, excetuando-se a notável queda dos teores no $\mathrm{T}_{2}$, no T. aestivum, houve tendência de decréscimo com o grau de hipoxia.

Por último e diferentemente de outros macronutrientes, os valores de magnésio encontrados foram muito próximos nas duas espécies, parecendo demonstrar não haver diferença na capacidade de extração e/ou exigência nutricional, entre as duas, reforçando também a coerência dos resultados com os obtidos por outros autores; além disso, também são coerentes com os observados por Letey et al. (1961) que apontam, para diferentes culturas, que a redução no acúmulo causado pela limitação de oxigeno no solo é na ordem de $\mathrm{K}>\mathrm{P}>(\mathrm{Ca}+\mathrm{Mg})$; já em relação aos micronutrientes no T. durum, o manganês, $\mathrm{o}$ zinco, o boro e o cobre apresentaram uma resposta negativa nítida quanto à intensidade de encharcamento; o mesmo decréscimo no T. aestivum foi observado somente para o boro havendo, em quase todos os outros, uma nítida tendência à discreta ou não responsividade, o que estaria em concordância com o apresentado por Glinski \& Stepiewski (1986) que afirmam que, dentre todos os micronutrientes, somente o manganês apresenta tendência geral em decrescer seu teor nas folhas e elevá-lo nas raízes. Considerando-se os valores do $T_{1}$ e da média dos tratamentos (excetuando-se o $\mathrm{T}_{4}$ ) verifica-se que no T. aestivum o teor foi de $62,8 \%$ em relação ao $T$. durum, inferindo-se ser este último muito mais exigente/extrativo em relação a esse elemento. Quanto ao ferro, o boro e o $\mathrm{Cu}$ (excetuando-se o $\mathrm{T}_{4}$ do $T$. durum) tanto a média dos tratamentos como os teores do $\mathrm{T}_{1}$ apontam para a mesma exigência ou extração desses micronutrientes nas duas espécies. $\mathrm{O}$ ferro, nas duas espécies, apresentou exatamente o comportamento esperado em resposta ao encharcamento, ou seja, embora discreto, um acréscimo gradativo do teor com o grau de intensidade de inundações. Infere-se ter sido isto causado pela intensidade de redução do $\mathrm{Fe}^{+++}$e sua maior disponibilidade no solo, cujo comportamento seria compatível com o do arroz. Segundo Glinski \& Stepiewski (1986) essa gramínea, que é bem adaptada ao encharcamento, mostra aumento em ferro nessas condições. Esta diagnose é importante pois um acúmulo excessivo causado pelo encharcamento pode trazer danos ao tecido, através da produção de radicais ativos (Crawford \& Braendle, 1996).

No T. aestivum o zinco apresentou tendência de não responsividade com o encharcamento, diferenciando-se apenas no $\mathrm{T}_{4}$. Por outro lado, a tendência de decréscimo com a hipoxia apresentada no $T$. durum, é coerente com o observado nessa mesma cultura, por Labanauskas et al. (1975).

Como consideração geral, a tendência do T. durum apresentar maiores teores dos macronutrientes na folha, com exceção do nitrogênio e do manganês, pode indicar maior adaptação do seu sistema radicular à hipoxia e/ou que é mais exigente que o $T$. aestivum, o que mereceria, então, uma adubação diferenciada. A diferenciação entre cultivares de trigo sob encharcamento no teor de nutriente na planta também foi observada por Huang et al. (1994) atribuindo-a uma absorção mais eficiente da cultivar tolerante em relação à suscetível; por outro lado, pode-se concluir, também, que a adubação foliar executada não atingiu o objetivo proposto ou, se contribuiu, o fez de forma insuficiente para suprir as deficiências nutricionais da planta, prejudicada pelo comprometimento da via radicular.

\section{Parâmetros físico-hídricos de monitoramento da hipoxia}

Imediatamente antes da aplicação do $\mathrm{TAH}$ no $\mathrm{T}_{4}$, aos 47 DAE, o LF estava a $13 \mathrm{~cm}$ de profundidade. A variação da taxa de difusão de oxigênio - TDO e do potencial redox ao longo do período de elevação, é apresentada na Figura 4.

(A)

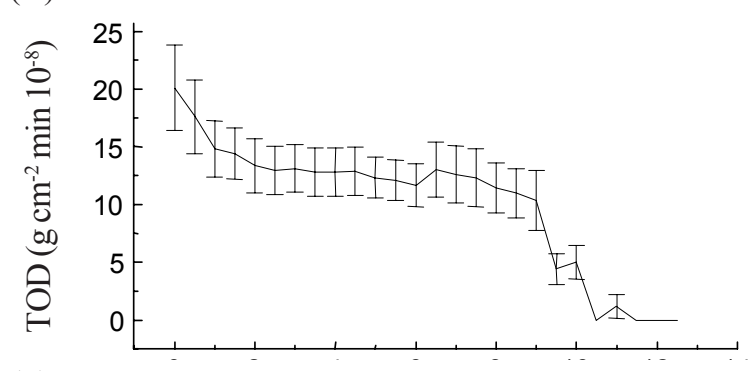

(B)

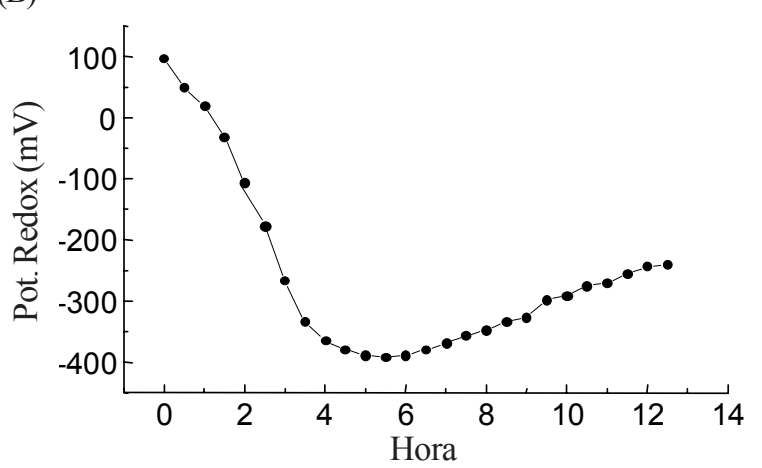

Figura 4. (A) Variação da TDO com média e erro-padrão, $n=4$; (B) Variação do potencial redox durante a elevação do LF no TAH do $\mathrm{T}_{4}$ 
Observa-se que, a $5 \mathrm{~cm}$ de profundidade, até mesmo anterior à elevação do LF, a TDO já era de $20,1.10^{-8} \mathrm{~g} \mathrm{~cm}^{-2} \mathrm{~min}^{-1}$. O valor obtido indicava, portanto, uma condição abaixo da crítica (Silverbush et al., 1979) atribuindo-se esse efeito à ação da franja capilar. Esta condição crítica fica ainda mais evidenciada se se considerar que, para plantas que se desenvolvem em condições naturais de áreas úmidas, portanto teoricamente bem adaptadas à hipoxia, foram apontados os limites entre $5 \mathrm{a} 25.10^{-8} \mathrm{~g} \mathrm{~cm}^{-2} \mathrm{~min}^{-1}$ (Poel, 1960). Esta condição altamente restritiva explica o comportamento nutricional da planta já discutido, e todas as inferências do comprometimento do sistema radicular. Stolzy \& Letey (1964) relacionando a TDO com a nutrição da planta, citam que, sob valores abaixo de 30 a $40.10^{-8} \mathrm{~g} \mathrm{~cm}^{-2} \mathrm{~min}^{-1}$, houve apenas ligeiros aumentos na concentração de $\mathrm{K}$ e $\mathrm{P}$ no girassol; apontam, também, que com Citrus sinensis, as concentrações de $\mathrm{P}, \mathrm{K}, \mathrm{Ca}, \mathrm{Mg}, \mathrm{Fe}, \mathrm{Mn}$ e B foram reduzidas em TDO abaixo de $33.10^{-8} \mathrm{~g} \mathrm{~cm}^{-2} \mathrm{~min}^{-1}$, comparadas com as concentrações observadas em plantas, desenvolvendo-se em TDO acima de $62.10^{-8} \mathrm{~g} \mathrm{~cm}^{-2} \mathrm{~min}^{-1}$.

O decréscimo do potencial redox foi mais brusco que da TDO e, após alcançar um ponto mínimo, elevou-se gradativamente, atingindo um ponto intermediário $(-240 \mathrm{mV})$ após 12,5 h (Figura 4B).

\section{Transformações fisiomorfológicas da planta}

A condição altamente restritiva caracterizada pela TDO e discutida antes, influi na bioquímica da planta, notadamente naquela relacionada ao sistema radicular. Alterações nas estruturas radiculares causadas pela ação do etileno podem condicionar enormemente o desempenho da planta. Como esperado, houve nítido e constante aumento da taxa de emissão de etileno com o grau de hipoxia, nas duas espécies (Figura 5). $\mathrm{O} \mathrm{T}_{4}$ apresentou-se, no T. aestivum, significativamente maior que o $\mathrm{T}_{1}$, enquanto no $T$. durum este mesmo tratamento foi significativamente maior que os $\mathrm{T}_{2}$ e $\mathrm{T}_{1}$. Deve-se ressaltar também, o comportamento coerente do $\mathrm{T}_{2}$ com tudo o que já foi discutido.

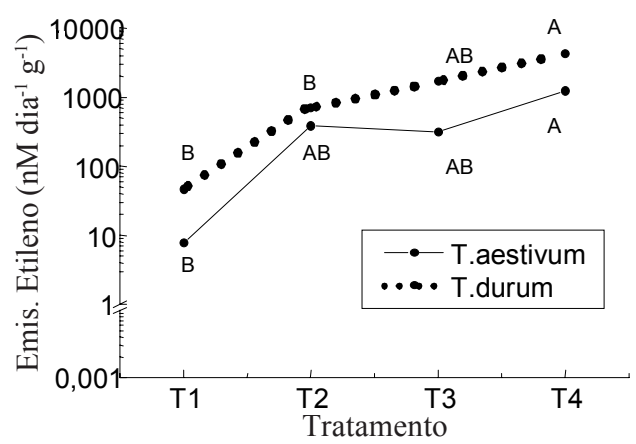

Figura 5. Avaliação do etileno. Signif. estatística $(\mathrm{P}<0,05)$; Duncan 5\%

Aos 75 DAE a análise do sistema radicular (Figura 6) revelou o crescimento de raízes adventícias nas plantas do $T_{2}$, ao contrário das conduzidas sob normoxia. Foram observadas algumas características típicas de raiz modificada, tais como mais porosas, superficiais e contando com uma mucilagem notável envolvendo toda a raiz. Sob condição mais drástica de hipoxia (Figura 6C) a planta já apresentava epiderme rompida, expondo cavidades com tecido poroso, que são, na realidade, tecidos
(A)

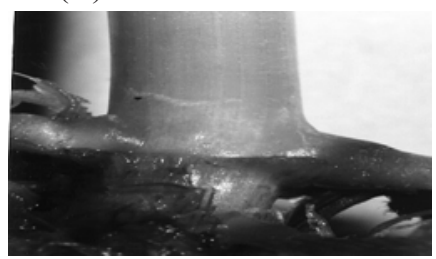

(C)

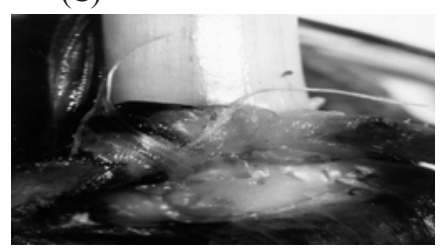

(B)

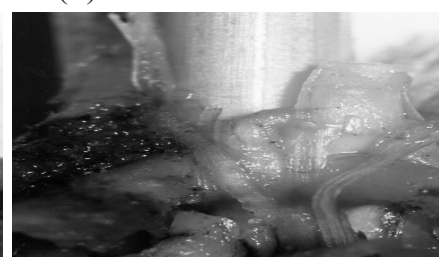

(D)

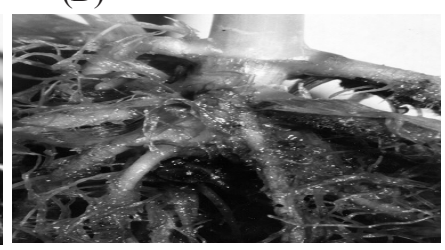

Figura 6. Transformações morfológicas em função do manejo do $L F$. (A) $\mathrm{T}_{1}$, (B) $\mathrm{T}_{2},(\mathrm{C}) \mathrm{T}_{4}$, (D) raízes adventícias no $\mathrm{T}_{2}$

facilitadores de penetração de $\mathrm{O}_{2}$ (Kawase, 1981) além de raízes fortemente necrosadas. A alta produção de raízes adventícias no $T_{2}$ é coerente com a avaliação do etileno que, embora não estatisticamente significativa em relação ao $\mathrm{T}_{1}$, nas duas espécies, sua curva aponta acentuado acréscimo no seu teor, notadamente no T. aestivum.

\section{Parâmetros biométricos de colheita}

Analisando-se as datas de colheita observa-se, tanto no T. aestivum como no T. durum, aceleração no ciclo com o estresse hipóxico, contrariando a tendência que vinha sendo observada ao longo do ciclo pelo fato de que, durante o desenvolvimento vegetativo a hipoxia retardou o ciclo e, a partir do florescimento, parece ter forçado a planta a acelerar a maturação. Segundo Glinski \& Stepniewski (1986) a tolerância das plantas ao encharcamento é função, dentre outros, do estádio cultural em que foi implantada a hipoxia. No caso de cereais, como o trigo, o florescimento é um dos mais sensíveis ao encharcamento. As considerações acima servem para explicar as mesmas datas de fechamento de ciclo dos tratamentos $\mathrm{T}_{3}$ e $\mathrm{T}_{4}$ e entre as duas espécies; já no $\mathrm{T}_{2}$, talvez em função de uma condição mais amena, houve ainda forte influência da característica genética.

Como comentário geral para as duas espécies, os parâmetros biométricos apresentaram gradiente negativo com a intensidade de hipoxia. As produtividades do $\mathrm{T}_{2}$, pouco menos da metade do $T_{1}$ mostram que, por um lado, foi significativo o prejuízo metabólico causado pela presença constante do LF a $15 \mathrm{~cm}$ e, por outro, situando-se a $30 \%$ acima da média nacional, referenda um potencial para esse tipo de estudo.

No T. aestivum houve manifestações fisiomorfológicas típicas de plantas de desenvolvimento suprimido. A tendência de acréscimo da altura de plantas (Tabela 3 ) do $\mathrm{T}_{3}$ em relação ao $\mathrm{T}_{2}$, conjugada a um comportamento inversamente proporcional na massa da matéria seca (significativo $\mathrm{P}<0,05$ ) aponta para um estiolamento no $\mathrm{T}_{3}$, já no $\mathrm{T}_{4}$, a altura e a MMS atingiram o menor valor, traduzindo uma nítida limitação no desenvolvimento. Apesar de não ter havido vantagem estatística do $\mathrm{T}_{2}$ em relação ao $\mathrm{T}_{3}$ nos demais componentes de produção, a MMS foi determinante para o rendimento de grãos ser significativamente distinto $(\mathrm{P}<0,05)$. Pode-se inferir que tanto a TAH como o momento em que ela foi aplicada no $\mathrm{T}_{3}$ foram a causa do comprometimento da MMS, bem como atestam o número de grãos por espigueta, grãos por espiga e massa de mil sementes, 
Tabela 3. Parâmetros ${ }^{1}$ biométricos do T. aestivum e do T. durum

\begin{tabular}{|c|c|c|c|c|c|c|c|c|c|}
\hline TRAT & $\begin{array}{l}\text { MMT } \\
\mathrm{kg} \mathrm{m}^{-1}\end{array}$ & $\begin{array}{c}\text { MMS } \\
\text { g }\end{array}$ & $\begin{array}{l}\mathrm{ALT} \\
\mathrm{cm}\end{array}$ & $\begin{array}{l}\mathrm{CE} \\
\mathrm{cm}\end{array}$ & $\overline{\mathrm{NE}}$ & $\overline{\mathrm{NGE}}$ & NGT & $\begin{array}{c}\text { DC } \\
\text { DAE }\end{array}$ & $\begin{array}{l}\text { REND } \\
\mathrm{kg} \mathrm{ha}^{-1}\end{array}$ \\
\hline \multicolumn{10}{|l|}{ T. aestivum } \\
\hline TRAT 1 & $0,45 \mathrm{~A}$ & $29,90 \mathrm{~A}$ & $74,54 \mathrm{~A}$ & 7,19 A & $17,09 \mathrm{~A}$ & $46,75 \mathrm{~A}$ & $2,74 \mathrm{~A}$ & 122 & $4.659,2 \mathrm{~A}$ \\
\hline TRAT 2 & $0,38 \mathrm{AB}$ & $19,22 \mathrm{~B}$ & $50,00 \mathrm{~B}$ & $6,76 \mathrm{~A}$ & $16,03 \mathrm{AB}$ & $34,10 \mathrm{~B}$ & $2,13 \mathrm{~B}$ & 119 & $2.170,2 \mathrm{~B}$ \\
\hline TRAT 3 & $0,34 \mathrm{~B}$ & $12,98 \mathrm{C}$ & $52,62 \mathrm{~B}$ & $7,15 \mathrm{~A}$ & $16,41 \mathrm{AB}$ & $32,20 \mathrm{~B}$ & $1,96 \mathrm{~B}$ & 115 & $947,3 \mathrm{C}$ \\
\hline TRAT 4 & $0,32 \mathrm{~B}$ & $12,51 \mathrm{C}$ & $49,73 \mathrm{~B}$ & $6,48 \mathrm{~A}$ & $14,60 \mathrm{~B}$ & $21,60 \mathrm{C}$ & $1,48 \mathrm{C}$ & 115 & $437,6 \mathrm{C}$ \\
\hline $\mathrm{F}$ & 7,2 & 103,0 & 25,9 & 1,7 & 3,3 & 20,3 & 21,4 & & 98,2 \\
\hline C.V. $(\%)$ & 13,1 & 9,7 & 9,3 & 8,5 & 8,1 & 15,2 & 12,0 & & 20,1 \\
\hline \multicolumn{10}{|l|}{ T. durum } \\
\hline TRAT 1 & $0,32 \mathrm{AB}$ & $23,07 \mathrm{~A}$ & $63,20 \mathrm{~A}$ & $5,91 \mathrm{~A}$ & $15,78 \mathrm{~A}$ & $49,96 \mathrm{~A}$ & 3,17 & 125 & $3.838,6 \mathrm{~A}$ \\
\hline TRAT 2 & $0,36 \mathrm{~A}$ & $18,26 \mathrm{~B}$ & $58,60 \mathrm{~A}$ & $6,07 \mathrm{~A}$ & $15,06 \mathrm{~A}$ & $33,72 \mathrm{~B}$ & 2,24 & 122 & $1.821,8 \mathrm{~B}$ \\
\hline TRAT 3 & $0,39 \mathrm{~A}$ & $11,22 \mathrm{C}$ & $44,00 \mathrm{~B}$ & $5,47 \mathrm{~A}$ & $14,31 \mathrm{~A}$ & $22,89 \mathrm{C}$ & 1,60 & 115 & $606,9 \mathrm{C}$ \\
\hline TRAT 4 & $0,27 \mathrm{~B}$ & $11,35 \mathrm{C}$ & $35,43 \mathrm{~B}$ & $6,04 \mathrm{~A}$ & $15,01 \mathrm{~A}$ & $17,02 \mathrm{C}$ & 1,13 & 115 & $449,9 \mathrm{C}$ \\
\hline $\mathrm{F}$ & $6,6^{*}$ & $117,0^{*}$ & $36,0^{*}$ & $1,6^{\mathrm{ns}}$ & $1,6^{\mathrm{ns}}$ & $67,6^{*}$ & & & $142,0^{*}$ \\
\hline C.V. $(\%)$ & 14,0 & 7,3 & 9,5 & 8,2 & 6,9 & 12,7 & & & 17,5 \\
\hline
\end{tabular}

${ }^{1}$ Médias seguidas da mesma letra não diferem, pelo teste de Duncan a $5 \% *$ Significativo a $5 \%$; (ns) não significativo

MMT - Massa da matéria seca; MMS - Massa de mil sementes; ALT - Altura da planta; CE - Comprimento da espiga; NE - Numero de Espigueta; NGE - Numero de grão por espiga; NGT - Numero de grão por espigueta; DC - Data de colheita; REND - Rendimento de grãos

parâmetros esses fortemente influenciados pelo desempenho da planta no florescimento/formação de grãos. No $\mathrm{T}_{3}$ e $\mathrm{T}_{4} \mathrm{O}$ estímulo adaptativo das PPAIs não visou à intensidade ou eficiência de que resultassem parâmetros biométricos de produção e rendimento de grãos aceitáveis.

Os três manejos induziram adaptações fisiomorfológicas tais, que permitiram a sobrevivência das plantas, mesmo sob encharcamento completo por mais de 50 dias, como no caso do $\mathrm{T}_{4}$. A título de comparação, Beard \& Martin (1970) afirmam que a Agrostis palustris Huds., a mais tolerante forrageira de três testadas, após 60 dias de continua submersão resultou em apenas $49 \%$ de sobrevivência sob água a temperatura de $20^{\circ} \mathrm{C}$, semelhante à deste experimento.

Concorreram negativamente as atípicas altas temperaturas no período e um reconhecido período talvez excessivamente longo de encharcamento contínuo no TAH, dificilmente observado na prática, em uma várzea dado ao normal refluxo hidrológico com o cessar do período chuvoso (recarga). Segundo Crawford \& Braendlle (1996) prolongada anoxia sob altas temperaturas esgota a reserva de carboidratos e permite o acúmulo de metabólitos causando danos ao tecido celular, quando reoxigenados. Não pode ser negligenciado o adicional efeito negativo da condição de água estagnada executada nesse experimento, facilmente evitada em condições naturais de várzeas. Segundo Davis \& Martin, citados por Beard \& Martin (1970) forrageiras submetidas a encharcamento morrem mais rapidamente sob condição de água estagnada que sob água corrente; já a comparação relativa entre os $\mathrm{T}_{3}$ e $\mathrm{T}_{4}$ leva a crer que a maior freqüência de PPAIs do $\mathrm{T}_{4}$ foi absolutamente prejudicial, baseando-se no fato de ter havido redução em todos os parâmetros biométricos. O perfeito entendimento sobre este processo pode ser a chave do sucesso na seleção de um manejo do LF mais adequado.

Um outro ponto pode estar ligado à bioquímica da planta. Concomitantemente com a maior freqüência de estresse hipóxico, ocorre também uma mais freqüente reoxigenação do solo, que pode vir a ser tão ou mais deletéria que a própria hipoxia em si. Pesquisas recentes apontam danos irreversíveis no tecido vegetal, quando retornam ao ar, havendo a formação de radicais ativos e produtos oxidativos tóxicos, como o acetaldeído (Crawford \& Braendlle, 1996).
Quanto ao T. durum, o gradiente dos parâmetros biométricos com o grau de hipoxia fica mais evidente. Permite-se apenas uma consideração à tendência do comprimento da espiga e do número de espigueta maior no $\mathrm{T}_{4}$ em relação ao $\mathrm{T}_{3}$ (não significativo $\mathrm{P}<0,05)$ sugerindo alguma possível tendência adaptativa. Esta vantagem, no entanto, perdeu sua importância com o decréscimo acentuado nos parâmetros biométricos desenvolvidos no período reprodutivo, quando da aplicação do TAH. A rigor, nenhuma outra consideração ou hipótese pode ser inferida diante da clara resposta do T. durum à hipoxia.

Os manejos implementados neste trabalho não permitiram indicar-se um potencial diferenciado ao cultivo sob encharcamento, a qualquer uma das espécies testadas.

\section{CONCLUSÕES}

1. A resposta diferenciada de exigência e/ou capacidade de extração entre as duas espécies, pode condicionar o sucesso produtivo do trigo sob hipoxia a estudos específicos, sobre suas exigências particulares e, conseqüentemente, a um plano de fertilização mais ajustado.

2. A adubação foliar foi ineficiente para suprir as deficiências nutricionais da planta decorrentes da limitação na absorção, via sistema radicular, causadas pela hipoxia no solo.

3. Os manejos com curtos períodos de hipoxia induziram a adaptações fisiomorfológicas tais, que permitiram a sobrevivência das plantas, mesmo sob encharcamento contínuo, por mais de 50 dias, como no caso do $\mathrm{T}_{4}$; essas, porém, não o foram na intensidade ou eficiência de que resultassem parâmetros de produção num nível adequado.

4. O manejo com o lençol freático mantido a $15 \mathrm{~cm}$ de profundidade durante todo o ciclo fenológico, foi o que propiciou melhor desempenho do trigo, em cultivo sob encharcamento.

\section{REFERÊNCIAS BIBLIOGRÁFICAS}

BEARD, J.B.; MARTIN, D.P. Influence of water temperature on submersion tolerance of four grasses. Agronomy Journal, Madison. v.62, n.2, p.257- 59, 1970. 
BRADFORD, K.J.; YANG, S.F. Physiological responses of plants to waterlogging. HortScience, Alexandria, v.16, n.1, p.25-30, feb. 1981.

CALHEIROS, R.O. Manejo da água de drenagem, objetivando adaptação fisiomorfológica de plantas mesófitas ao encharcamento. Piracicaba: ESALQ/USP, 2000, 127p. Tese Doutorado

CRAWFORD, R.M.M.; BRAENDLE, R. Oxigen deprivation stress in a changing environment. Journal of Experimental Botany, Oxford. v.47, n.295, p.145-9, 1996.

GARRITY, D.P.; PERNITO, R. Mungbean response to surface drainage when grown as a pre-rice crop on waterlog-prone ricelands. Agricultural Water Management, Amsterdam. v.29, n.3, p.299-314, 1996.

GLINSKI, J.; STEPNIEWSKI, W. Soil aeration and its role for plants. 2 ed. Florida: CRC Press Inc, 1986. 228p.

HERRMANN, P.S.P.; ANDRADE, M.G.; COLMAGO, L.A., SILVEIRA, P.M. Equipamento para medida da taxa de difusão de oxigênio e potencial de oxiredução no solo. Pesquisa Agropecuária Brasileira, Brasília. v.32, n.7, p.725-30, 1997.

HOOK, D.D.; BROWN, C.L. Root adaptation and relative flood tolerance of five gardwood species. Forest Science, Washington. v.19, n.3, p.225-29, 1973.

HUANG, B.R. JOHNSON, J.W.; NESMITH, S.; BRIDGES, D.C. Growth, physiological and anatomical responses of two wheat genotypes to waterlogging and nutrient supply. Journal of Experimental Botany,Oxford. v.45, n.271, p.193-202, 1994.

JOHNSON, J.; COBB, B.G.; DREW, M.C. Hypoxic induction of anoxia tolerance in roots of Zea mays, L. Plant Physiology, Rockville. v.1, p.873-41, 1989.

KAWASE, M. Anatomical and morphological adaptation of plants to waterlogging. HortScience, Alexandria. v.16, n.1, p.8-12.1981.

LABANAUSKAS, C.K.; STOLZY, L.H.; LUXMOORE, R.J. Soil temperature and soil aeration effects on concentration and total amounts of nutrients in "Yecora" wheat grain. Soil Science, Baltimore. v.120, n.6, p.450-54, 1975.

LETEY, J.; LUNT, O.R.; STOLZY, L.H.; SZUSKIEWCZ, T.E. Plant growth, water use and nutritional response to rhizosphere differentials of oxygen concentration. Soil Science Society America Proceedings. Madison. v.25, n.3, p.183-186, 1961.

MINCHIN, F.R.; SUMMERFIELD R.J. Symbiotic nitrogen fixation and vegetative growth of cowpea (Vigna unguiculata, $L$. Walp.) in waterlogged conditions. Plant and Soil, Hague. v.45, n.1,p.113-27, 1976.
POEL, L.N. A preliminary survey of soil aeration condition in a Scottish hill grazing. Journal of Ecology, Oxford. v.48, n.2, p.733-736, 1960.

SCHAFFER, B.; ANDERSEN, P.C.; PLETZ, R.C. Responses of fruits crops to flooding. In: JANICK, J. (ed.). Horticultural Reviews, New York: John Wiley \& Sons, Inc., 1992, v.13, p.257-313.

SENA GOMES, A.R.; KOZLOWSKI, T.T. Growth responses and adaptations of Fraxinus pennylvanica seedlings to flooding. Plant Physiology, Rockville. v.66, n.1, p.267-71, 1980.

SILVERBUSH, M.; GORNAT, B.; GOLDBERG, D. Effect of irrigation from a point source (trickling) on oxygen flux and on root extension in the soil. Plant and Soil, Hague. v.52, n.4, p.507-514, 1979 .

SINGH, B.P.; TUCKER, K.A.; SUTTON, J.D.; BHARDWAJ, H.L. Flooding reduces gas exchange and growth in snap bean. HortScience, Alexandria. v.26, n.4, p.372-73, 1991.

SOJKA, R.E.; STOLZY, L.H. Soil-oxygen effects on stomatal response. Soil Science, Baltimore. v.130, n.6, p.350-58, 1980.

STOLZY, L.H.; LETEY, J. III. Correlation of plant response to soil oxygen diffusion rates. HILGARDIA, Oakland. v.35, n.20, p.567-76, 1964.

TROUGHT, M.C.T.; DREW, M.C. The development of waterlogging damage in wheat seedlings (Triticum aestivum $L$. ). II Accumulation and redistribution of nutrients by the shoot. Plant and Soil, Hague. v.56, n.2, p.187-99. 1980.

UMAHARAN, P.; ARIYANAYAGAM, R.P.; HAQUE, S.Q. Effect of short-term waterlogging applied at various growth phases on growth, development and yield in vigna unguiculata. Journal of Agricultural Science, Cambridge. v.128, n.2, p.189-98, 1997.

van WADMAN, S.H.; van ANDEL, O.M. Interdependence of growth, water relations and abscisic acid level in Phaseolus vulgaris during waterlogging. Physiologia Plantarum, Copenhagen. v.63, n.2, p.215-20, 1985.

WATERS, I.; MORRELL, S.; GREENWAY, H.; COLMER, T.D. Effects of anoxia on wheat seedlings. II. Influence of $\mathrm{O}_{2}$ supply prior to anoxia on tolerance to anoxia, alcoholic fermentation, and sugar levels. Journal of Experimental Botany, Oxford. v.42, n.244, p.1437-47, 1991.

WHITE, W.J.; MOLANO, C.H. Production of common bean under saturated soil culture. Field Crops Research, Amsterdan. v.36, n.1, p.53-8, 1994.

WIGNARAJAH, K.; GREENWAY, H. Effect of anaerobiosis on activities of alcohol dehidrogenase and pyruvate decarboxylase in roots of Zea mays, L. New Phytologist, Oxford. v. 77, n., p.575-84, 1976. 